\title{
Measurements on Passive Tunable Optical Filters for DWDM
}

\author{
Carmen Vázquez, Salvador Vargas, José Manuel S. Pena \\ Depto. de Tecnología Electrónica, Escuela Politécnica. Superior, Universidad Carlos III, Av. \\ Universidad 30, Leganés 28911, Madrid, E-mail: cvazquez@ing.uc3m.es \\ Pedro Corredera \\ Centro de Tecnologías Físicas, "Leonardo Torres Quevedo", Instituto de Física Aplicada, CSIC, \\ C/ Serrano 144, 28006, Madrid, Spain
}

\begin{abstract}
Measurements on a passive optical fibre tunable filter are presented. The device is based on a loop mirror in a ring resonator. The loop mirror allows tuning by changing the coupling coefficient of a coupler. The novel filter structure allows planar optical integration for having higher free spectral ranges. Novel and simple design equations for the filter parameters and the tuning process are reported. There is a good agreement between measurements and theory.
\end{abstract}

\section{INTRODUCTION}

The need for increasing bandwidth has driven standard institutions to extend the transmission windows from the conventional $\mathrm{C}$ band to $\mathrm{O}, \mathrm{E}, \mathrm{L}, \mathrm{S}, \mathrm{U}$ bands and/or to reduce the channel spacing. This last technique is used in the DWDM (Dense Wavelength Division Multiplexing) systems with carriers spacing of $50 \mathrm{GHz}$, or below [1]. These more dense spectra need filters able to separate very narrow channels with high crosstalk levels. Another interesting approach is having filter structures easily translated to an integrated optic implementation with a high level of integration. In this respect different optical filters have been developed [2,3] using as building blocks, Mach Zendher (MZ) interferometers and recirculating delay lines with phase shifters or amplified ring resonators [4,5]. In this paper, we present a novel tunable filter, based on the structure reported in [5] but in its passive form to operate as a notch filter. Design equations are included along with measurements, on a fibre optic prototype, showing the tuning capability of the device.

\section{FILTER DESIGN}

Passive optical filter architecture is shown in Figure 1. It is made of optical directional couplers and a multi-reflection function in a ring resonator. More specifically, a loop is used to implement this multi-reflection function. The transfer function of the device is defined as $\mathrm{P} 3 / \mathrm{P} 1$. Filter design equations are derived using the $\mathrm{z}$-transform technique to allow for a simple location of poles and zeroes of the transfer function. 


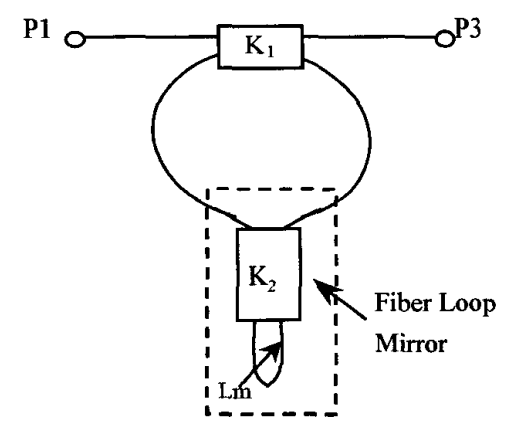

Figure 1: Schematic of the device

The transmission and reflection function of the loop, FT and FR respectively, are given by:

$$
\begin{aligned}
& F T=\left(1-\gamma_{2}\right)\left(1-2 K_{2}\right) e^{-(\alpha+j \beta) L m} \\
& F R=2 j\left(1-\gamma_{2}\right)\left(K_{2}\left(1-K_{2}\right)\right) e^{-(\alpha+j \beta) L m}
\end{aligned}
$$

where $\mathrm{K}_{2}$ and $\gamma_{2}$ are the loss and coupling coefficient of the coupler used to connect the loop to the ring as shown in Figure 1, Lm is the loop length, $\alpha$ is the attenuation coefficient and $\beta$ the propagation constant.

Using equations $1-2$ and the relations between electrical fields in the ring [5,6], the following expression for the output power transfer function are obtained:

$$
\frac{P_{3}}{P_{1}}=\left(1-\gamma_{1}\right)^{1 / 2}\left[\frac{\left(1-K_{1}\right)^{1 / 2}-\left(\left(1-\gamma_{1}\right) L^{*}\right)^{1 / 2}\left(2-K_{1}\right) F T \cdot Z^{-1}+\left(1-\gamma_{1}\right) L^{*}\left(1-K_{1}\right)^{1 / 2}\left(F T^{2}-F R^{2}\right) Z^{-2}}{\left(1-Z p_{1} Z^{-1}\right)\left(1-Z p_{2} Z^{-1}\right)}\right]
$$

where $\gamma_{1}$ and $K_{1}$ are the loss and coupling coefficient of the input/output coupler. $Z=e^{j \beta(L r+L m)}$, with $L r$ the ring length. $L^{*}=L \cdot e^{-\alpha(L r+L m)}$ with $L$ any loss in the ring apart from propagation loss, such as connectors and splices losses.

The zeroes and the modulus of the poles of this transfer function are given by:

$$
\begin{aligned}
& \left|Z p_{1,2}\right|=\left(\left(1-\gamma_{1}\right)\left(1-K_{1}\right) L^{*}\right)^{1 / 2}\left(1-\gamma_{2}\right) \\
& Z c_{1}=\frac{\left(1-\gamma_{1}\right) L^{*}\left\lfloor\left(2-K_{1}\right) F T+\sqrt{F T^{2} K_{1}^{2}+4 F R^{2}\left(1-K_{1}\right)}\right]}{2\left(1-K_{1}\right)^{1 / 2}} \\
& Z c_{2}=\frac{\left(1-\gamma_{1}\right) L^{*}\left\lfloor\left(2-K_{1}\right) F T-\sqrt{F T^{2} K_{1}^{2}+4 F R^{2}\left(1-K_{1}\right)}\right]}{2\left(1-K_{1}\right)^{1 / 2}}
\end{aligned}
$$


Equations 5 and 6 reveals that the zeroes are complex conjugate if $\mathrm{K} 1<$ Kcritic. This Kcritic depends on K2. If that condition is fulfilled the modulus and phase of the zeroes are given by:

$$
\begin{aligned}
& \left|Z c_{1,2}\right|=\left(1-\gamma_{1}\right)^{1 / 2}\left(1-\gamma_{2}\right) L^{*} \\
& \phi_{c 1}=\tan ^{-1}\left[\frac{\sqrt{K_{1}^{2}\left(1-2 K_{2}\right)^{2}-16\left(1-K_{1}\right)\left(1-K_{2}\right) K_{2}}}{\left(2-K_{1}\right)\left(1-2 K_{2}\right)}\right]
\end{aligned}
$$

The phase depends on $\mathrm{K} 1$ and $\mathrm{K} 2$ so we can use both parameters to select the minima location, that is, for tuning the filter response.

In designing the passive optical filter as a notch filter, it is important to have a transfer function with a dominant effect of the zeros against the poles. From that design criteria we can delimit a set of possible values for the filter parameters such as coupling coefficients and losses. A practical tool to verify that condition is the zero and pole gain $(\mathrm{Gc}, \mathrm{Gp})$ defined as the gain/loss value ( $\mathrm{L}^{*}$ in equations 4 and 7) to have the modulus of the zeros and poles equal to unity. In Figure 2, we have plotted $\mathrm{Gc}$ and $\mathrm{Gp}$ versus $\mathrm{K} 1$ with $\mathrm{K} 2$ as a parameter. We see

that for each $\mathrm{K} 2$ there is a range of $\mathrm{K} 1$ values where the zeroes are complex

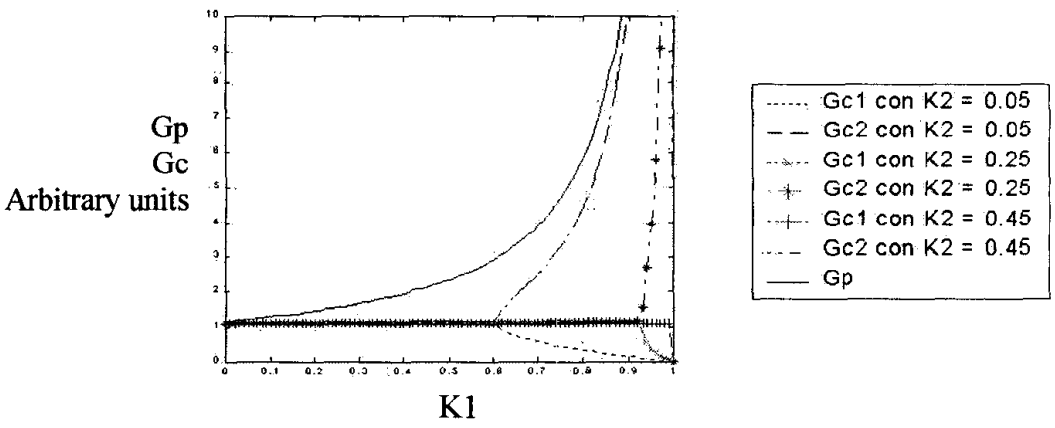

Figure 2: Pole and zero gains. $\gamma_{1}=\gamma_{2}=0.05$

conjugated, so $\left|z_{c 1}\right|=\left|z_{c 2}\right|$ that implies that $\mathrm{Gc1}=\mathrm{Gc} 2=\mathrm{Gc}$, and the filter can be tuned. Selecting K2 close to 0.5 the tuning range is increased. Stable notch filters with high crosstalk are obtained in the region below Gc.

On the other hand, from Figure 2 we select $\mathrm{K} 1$ around 0.9 to have the zeroes effect dominating on the poles for a passive filter. This design is a very stable filter because the poles are far away from the unit circle.

\section{EXPERIMENTAL SET-UP AND MEASUREMENTS}

In order to show the utility and the behaviour of the novel passive optical filter, we have measured the output power for different $\mathrm{K} 2$ values. A filter using optical 
fibre technology is implemented because it was simpler for us to do so. Although, to have a greater Free Spectral Range (FSR), integrated optical filters could be developed. In the following we are going to describe the experimental set-up and the measurements we have taken.

\subsection{Experimental set-up}

In Figure 3, it can be seen the set-up that it has been used in the measurements. A tunable laser PHOTONETICS TUNICS PLUS-L $(1510-1610 \mathrm{~nm})$ at $1550 \mathrm{~nm}$, with a $150 \mathrm{KHz}$ linewidth and a side mode suppression ratio $>45 \mathrm{~dB}$ is used. To avoid temperature and vibrations effects among others, due to the coherence nature of the measurements, we have externally modulated the laser with a RF signal through a Mach-Zenhder Electro-Optic Modulator (EOM-MZ) from UTP that modulates up to $4 \mathrm{GHz}$. The RF signal comes from a network analyser, HP8510B, which sweeps a frequency window of $66 \mathrm{MHz}$, with 201 measurement points equally spaced, at a centre frequency of $1 \mathrm{GHz}$. The optical detection is carried out by an optical-to-electrical converter (OE), TEKTRONICS OE-502, which consists of a Ge APD and a 40dB attenuator. From $\mathrm{OE}$ output the network analyser measures the output power amplitude and phase. Splices are used instead of connectors to avoid additional resonator perturbations in the measurements.

\subsection{Measurements}

As previously mentioned, our objective is to verify the tuning capability of our optical filter. In doing so, using Figure 2 and equations 4-8, we see that K2 modifies the zeroes location once $\mathrm{Kl}$ is fixed. So we measure the filter response

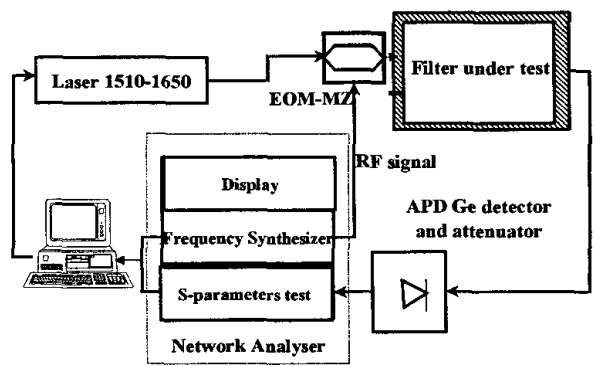

Figure 3: Experimental set-up

for different $\mathrm{K} 2$ values as it is shown in Figures 4,5 and 6. The filter is made using $2 \mathrm{X} 2$ monomode fibre couplers with $1 \mathrm{~m}$ of pigtail in each port. Each measurement has been taken several times and the results of all of them are simultaneously plotted in Figures 4.2, 5.2 and 6.2, showing a good stability. In changing K2 value, different couplers have been used, so new splices have been developed in each measurement, causing slightly different losses. The parameters of the measured filter are $\mathrm{K} 1=0.92, \gamma_{1}=0.065$ and $\mathrm{L}=0.6,0.6$ and $0.5, \gamma_{2}=0.06$, 0.07 and 0.07 , for Figures 4.2, 5.2 and 6.2 respectively. 

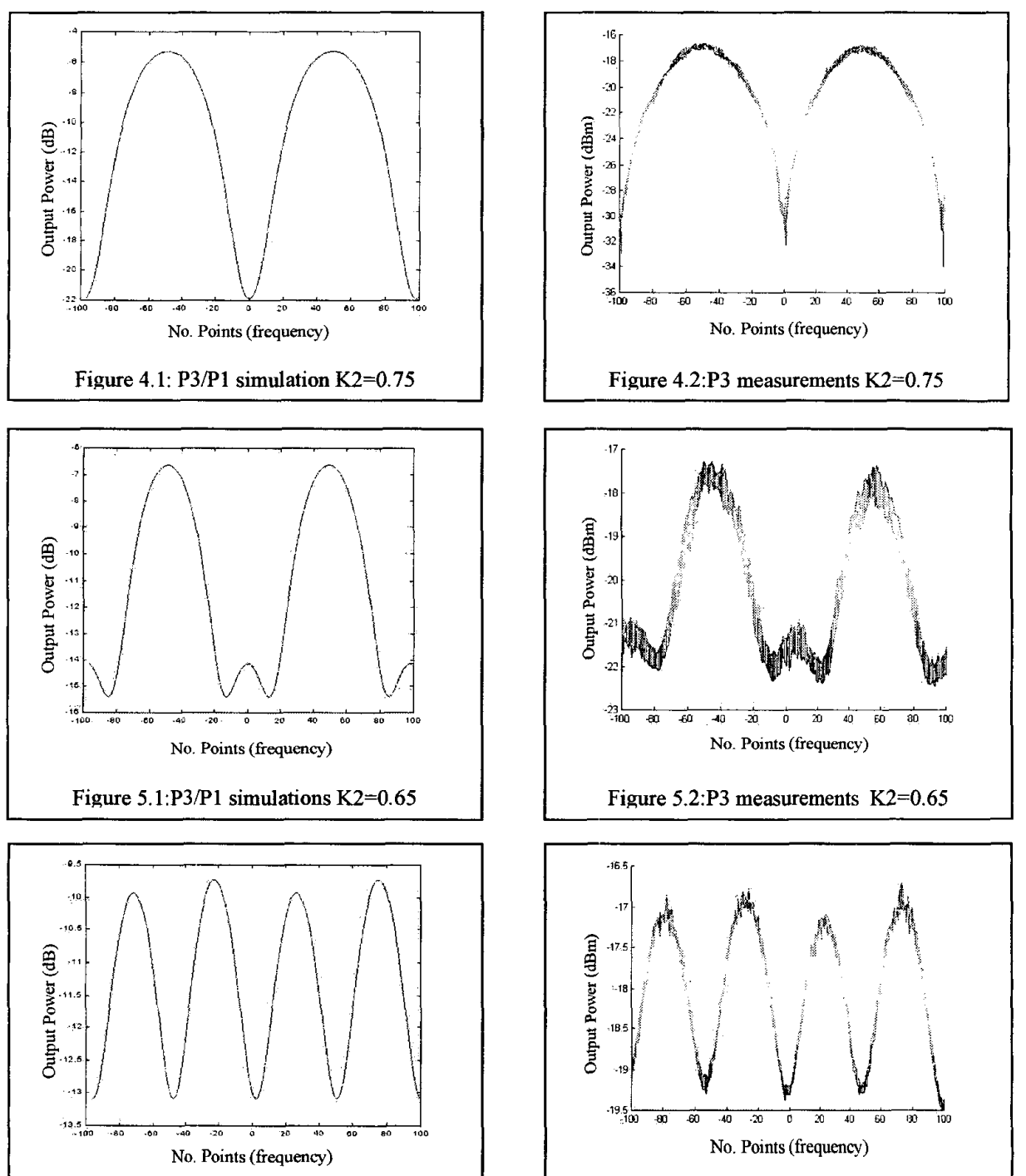

Figure 6.1:P3/P1 simulation $\mathrm{K} 2=.505$

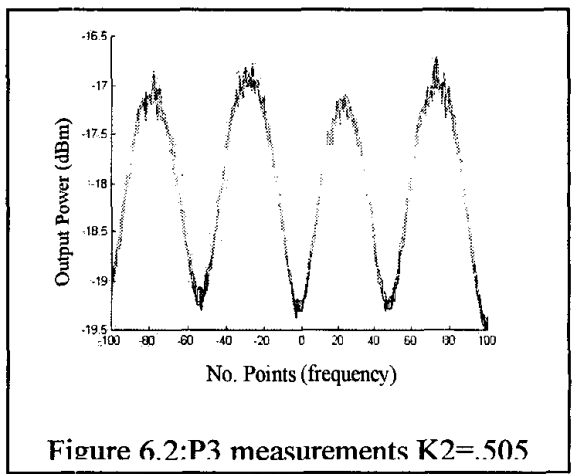

As expected, we can see how the minima are shifted in one FSR $(33 \mathrm{MHz})$, with the shortest separation for $\mathrm{K} 2=0.505$, increasing for $\mathrm{K} 2=0.65$ and almost doubling for $\mathrm{K} 2=0.75$. There is a good agreement between measurements and theory. As we have not used any device to control the losses, the zeroes are not very close to the unit circle so the largest crosstalk measured is of $16 \mathrm{~dB}$. 


\section{CONCLUSIONS}

A passive notch filter is designed using a novel configuration made of a fibre loop in a ring resonator. The multi-reflections originated by the loop are controlled through a coupling coefficient; which is responsible of the tuning process. Novel simple equations for designing and tuning are reported. A passive notch filter is implemented using fibre optic technology. The structure can be developed using planar optical integration. Measurements for different coupling coefficients are reported to verify the tuning characteristics of the device and to test the theory. Higher crosstalk on the measurements can be obtained through a proper control of the losses in the ring resonator.

\section{ACKNOLEDGEMENT}

This work was supported by Comunidad Autónoma de Madrid (CAM-07T-00112001). $\mathbf{M}^{\mathrm{a}}$ Luisa Hernanz has helped in optimising the set-up.

\section{REFERENCES}

[1] A. Farbert, G. Mohs, S. Spalter, J.-P. Elbers, C. Furst, A. Schopflin, E. Gottwald, C. Scheerer, C. Glingener, "7 Thb/s bidirectional interleaved transmission with $50 \mathrm{GHZ}$ channel spacing", ECOC'00, PDP-3, 2000.

[2] N. Ngo, and L. N. Binh, "Novel Realization of Monotonic Butterworth-type Lowpasss, Highpass, and Bandpass Optical Filters Using Phase-Modulated Fiber-Optic Interferometers and Ring Resonators", J. Lightwave Tech., vol. 12, pp. 827-840, 1994.

[3] C. K. Madsen, "General IIR Optical Filter Design for WDM Applications Using All-Pass Filters", J. Lightwave Tech., vol. 18, 860-868, 2000.

[4] C. Vázquez, S. Vargas, J. M. S. Pena. "Design and Tolerance Analysis of a Router using an Amplified Ring Resonator and Bragg Gratings", Applied Optics, vol. 39, pp. 1934-1940, 2000.

[5] S. Vargas, C. Vázquez, J. M. S. Pena. "Novel tunable optical filter employing a fiber loop mirror for synthesis applications in WDM", LEOS'01. vol. II, 899-900, 2001.

[6] Jianluo Zhang, John W. Y. Lit, "Compound fiber ring resonator: theory", $J$. Opt. Soc. Am. A, vol. 11, pp. 1867-1873, 1994. 